\title{
Induction of apoptosis by cytoplasmically localized wild-type p53 and the S121F mutant super p53
}

\author{
KATSUHIRO YASUDA, SHUNSUKE KATO, YASUHIRO SAKAMOTO, GOU WATANABE, \\ SATSUKI MASHIKO, ATSUKO SATO, YUICHI KAKUDO and CHIKASHI ISHIOKA \\ Department of Clinical Oncology, Institute of Development, Aging and Cancer, \\ Tohoku University, Sendai, Miyagi 980-8575, Japan
}

Received February 13, 2012; Accepted February 21, 2012

DOI: $10.3892 / 01.2012 .624$

\begin{abstract}
After DNA damage, p53 is accumulated in the nucleus and transactivates downstream genes and induces apoptosis. There are two pathways in p53-dependent apoptosis, the transactivation-dependent and -independent pathway. In this study, we constructed p53-inducible glioblastoma cell lines and analyzed them for the induction of apoptosis and transactivation of p53-downstream genes after the nuclear or cytoplasmic expression of p53. To sequester p53 in the cytoplasm, we used p53 mutant with arginine to glycine substitution at residue 306 (R306G). Wild-type p53 retained the ability to arrest the cell cycle, and a p53 mutant with serine to phenylalanine substitution at residue $121(\mathrm{~S} 121 \mathrm{~F})$, which has a strong ability to induce apoptosis, retained this ability even when both the wild-type and p53 and S121F mutant were exclusively sequestered from the nucleus into the cytoplasm. Notably, cytoplasmically sequestered wild-type p53 and S121F mutant transactivated the downstream genes with distinct expression profiles, and the strong apoptotic ability of S121F was not associated with its transactivation activity. These results underscore the existence of transactivation-independent apoptosis and cytoplasmic function of $\mathrm{p} 53$.
\end{abstract}

\section{Introduction}

TP53 tumor suppressor gene is one of the most commonly mutated genes in human neoplasia, and approximately $80 \%$ of these mutations are missense mutations $(1,2)$. The gene product, $\mathrm{p} 53$ protein, is a nuclear transcriptional activator that is activated by post-translational modification, including phosphorylation and acetylation, in response to DNA-damaging stresses. Activated p53 is stabilized, accumulates in the nucleus and binds to p53-responsive elements (p53REs) in the promoter

Correspondence to: Dr Chikashi Ishioka, Department of Clinical Oncology, Institute of Development, Aging and Cancer, Tohoku University, Sendai, Miyagi 980-8575, Japan

E-mail: chikashi@idac.tohoku.ac.jp

Key words: p53, tumor suppressor, apoptosis, subcellular localization region of p53-downstream genes (3). Transactivation of these genes, including $p 21 W A F 1, M D M 2, p 53 A I P 1, B A X, N O X A$ and $P U M A$, results in cell cycle arrest and apoptosis.

Most p53 mutants with a single amino acid substitution found in human neoplasm lose the ability to bind to p53REs, and this functional defect is thought to be one of the most important oncogenic events caused by TP53 mutation (4). Therefore, the translocation of p53 into the nucleus is crucial for normal p53 function. Cytoplasmic sequestration of wild-type p53 was observed in undifferentiated neuroblastoma, breast cancer, retinoblastoma, colorectal carcinoma and glioblastoma cells (5-7). In all these cells, wild-type p53 is inactivated since it is retained in the cytoplasm. Although the precise mechanism underlying the cytoplasmic sequestration remains unclear, several molecular mechanisms have been proposed: i) a mutation in the bipartite sequence of p53 (residues 305 and 306) (8) or a truncated mutation of the nuclear localization motif receptor protein importin- $\alpha$ (9); ii) hyperactive nuclear export by an MDM2-dependent pathway (10); and iii) overexpression of cytoplasmic tethering proteins, such as mortalin (11), cullin 7 (12) and PARC (13). The mutations in the bipartite sequence have been analyzed comprehensively, and these mutants were shown to lose transactivation activity in a yeast functional assay (14).

In contrast to tumor-derived loss-of-function p53 mutants, other types of p53 mutants (super p53s) have a stronger ability to induce apoptosis than wild-type p53. Among these, a p53 mutant with a serine to phenylalanine substitution at residue 121 (S121F) has a distinct affinity to bind p53REs from wild-type p53 (15). S121F induces a more potent apoptosis than wild-type p53 in mammalian cell lines. The transcriptional activity of $\mathrm{S} 121 \mathrm{~F}$ for downstream genes, however, is less efficient than that of wild-type p53 (16). In addition, different expression profiles among super p53s have been reported (17). These results suggest that transactivation-independent cytoplasmic activity occurs in p53-dependent apoptosis and that S121F may be a diverged mutant with enhanced cytoplasmic activity.

To test this hypothesis, we expressed wild-type and S121F p53 in the nucleus or cytoplasm of p53-null SF126 glioblastoma cells using a p53 mutant with an arginine to glycine substitution at residue 306 (R306G), and analyzed them for induction of apoptosis and transactivation of p53-downstream genes following the p53 induction. 


\section{Materials and methods}

Construction of stable SF 126 glioblastoma cell lines. The plasmids pCR259-WTp53, pCR259-S121F and pCR259-R306G were previously constructed (14). pCR259-S121F-R306G was constructed by inserting a small fragment of pCR259-R306G into the Bsu36I/EagI site of pCR259-S121F. The small NheI/ EagI fragments of the four pCR259-based plasmids were inserted into the NheI/NotI site of pcDNA5/TON (Invitrogen, Carlsbad, CA, USA). The resulting plasmids were designated pcDNA5/TON-WTp53, pcDNA5/TON-S121F, pcDNA5/ TON-R306G and pcDNA5/TON-S121F-R306G, respectively. The stable SF126 cell lines expressing tetracycline-inducible p53 were constructed according to the protocol described in the T-Rex ${ }^{\mathrm{TM}}$ System (Invitrogen) using the four pcDNA5/ TON-based plasmids. For each category, several stable clones were selected by hygromycin B $(100 \mu \mathrm{g} / \mathrm{ml})$ and two independent stable clones were used.

Western blot analysis. The cell lines were harvested and the cells were resuspended in lysis buffer containing $50 \mathrm{mM}$ Tris- $\mathrm{HCl}, \mathrm{pH}$ 8.0, $150 \mathrm{mM} \mathrm{NaCl}, 5 \mathrm{mM}$ EDTA and 1\% protease inhibitor cocktail (Sigma-Aldrich, St. Louis, MO, USA). Cell lysates were centrifuged for $10 \mathrm{~min}$ at $4^{\circ} \mathrm{C}$. The supernatants were resolved by SDS-PAGE and transferred to PVDF membranes. The membranes were incubated with anti-p53 (FL-393; Santa Cruz Biotechnology, Santa Cruz, CA, USA) and mouse anti-actin (Sigma-Aldrich), followed by incubation with goat anti-rabbit Alexa Fluor $680 \mathrm{IgG}$ (Invitrogen) and goat anti-mouse IR Dye $800 \mathrm{CW}$ IgG (Rockland, Gilbertsville, PA, USA). Expression of both p53 and $\beta$-actin was visualized using an Odyssey Infrated Imaging System (LI-COR, Lincoln, NE, USA).

Immunofluorescent analysis. Each cell line was cultured on poly-D lysine-coated Lab-Tek Chamber Slides ${ }^{\mathrm{TM}}$ (Nalge Nunc, Rochester, NY, USA) until $70 \%$ confluence was achieved. At $24 \mathrm{~h}$ after the addition of $10 \mathrm{ng} / \mathrm{ml}$ doxycycline or phosphate-buffered solution (PBS), the cells were fixed with acetone-methanol (1:1) and incubated for $20 \mathrm{~min}$ at $-20^{\circ} \mathrm{C}$. After washing with PBS and blocking with 5\% non-fat milk in PBS containing $0.05 \%$ Tween-20 for $1 \mathrm{~h}$, cells were incubated with FITC-conjugated mouse anti-p53 (DO-1 FITC; Santa Cruz Biotechnology, Santa Cruz, CA, USA) diluted at 1:500, stained with propidium iodide and then visualized on an LSM5 PASCAL (Carl Zeiss, Jena, Germany).

Cell proliferation assay. Cells $\left(4 \times 10^{3}\right)$ were seeded and incubated in a 96-well plate for $24 \mathrm{~h}$. Doxycycline $(10 \mathrm{ng} / \mathrm{ml})$ or PBS was added to the medium and the cells were then cultured until $72 \mathrm{~h}$ at $37^{\circ} \mathrm{C}$. At $0,24,48$ and $72 \mathrm{~h}$ after the addition of doxycycline, $10 \mu \mathrm{l}$ of the Cell Counting Kit-8 (Dojin Laboratories, Kumamoto, Japan) was added to each well and the cells were incubated for $2 \mathrm{~h}$ at $37^{\circ} \mathrm{C}$. Absorbance at $490 \mathrm{~nm}$ was read with a microplate reader. Each data point is derived from triplicate experiments. The absorbance values at 24,48 and $72 \mathrm{~h}$ were normalized by the value at $0 \mathrm{~h}$.

Cell cycle analysis by fluorescence-activated cell sorting. Cells $\left(1 \times 10^{6}\right)$ were seeded and incubated in a $10-\mathrm{cm}$ culture plate for $24 \mathrm{~h}$, and then incubated in the presence of doxycycline $(10 \mathrm{ng} / \mathrm{ml})$. After $24 \mathrm{~h}$, the cells were collected and stained with propidium iodide $(50 \mu \mathrm{g} / \mathrm{ml})$. The stained cells were filtered through 50- $\mu \mathrm{m}$ nylon mesh and analyzed using a Cytomics FC500 (Beckman Coulter, Miami, FL, USA). The subfraction of cells in each phase of the cell cycle was calculated using Multicycle software (Phoenix Flow Systems, San Diego, CA, USA). The average subfraction value of two independent cell lines was calculated.

Quantitative real-time PCR analysis. Total RNA was extracted from cells in the presence or absence of doxycycline using an RNeasy Mini kit (Qiagen, Gaithersburg, MD, USA). RNA $(1 \mu \mathrm{g})$ was converted to cDNA using a High-Capacity cDNA Reverse Transcription kit (Applied Biosystems, Foster City, CA, USA) with random hexamers. TaqMan Gene Expression Assay was performed on the ABI 7500 real-time PCR System (Applied Biosystems) according to the manufacturer's protocol. Assay ID was as follows; GAPDH,Hs99999905_m1; ACTB ( $\beta$-actin), Hs99999903_m1; MDM2, Hs01066938_m1; p21 (CDKN1A), Hs00355782_m1; BAX, Hs00180269_m1; NOXA (PMAIP1), Hs00560402_m1; PUMA (BBC3), Hs00248075_m1; and P53AIP1, Hs00223141_m1. The expression level of each p53 target gene was collected by either $\beta$-actin or GAPDH (data not shown). A relatively induced expression was measured as a ratio of the collected value of doxycycline presence against that of doxycycline absence. Two independent clones were analyzed, and the data were shown as a mean of four replicates with an error bar of standard deviation.

\section{Results}

Cytoplasmic sequestration of p53 by R306G mutation. To examine the cytoplasmic activity of wild-type p53 and the S121F mutant, we constructed a series of stable SF126 glioblastoma cell lines. These cells expressed wild-type p53, S121F, R306G or S121F-R306G double mutants in the presence of doxycycline (Fig. 1). To examine the cellular localization of doxycycline-induced p53, an immunofluorescent analysis was performed (Figs. 1 and 2). Both wild-type p53 and S121F localized mostly to the nucleus. In the presence of the R306G mutation in the same p53 molecule, wild-type p53 and S121F were exclusively sequestered from the nucleus to the cytoplasm. To quantify the degree of sequestration of p53, 100 cells of each cell clone were scored as having nuclear, cytoplasmic or both nuclear and cytoplasmic patterns (Fig. 2). Both wild-type and S121F thoroughly localized in the nucleus ( $>95 \%$ of cells), and no cell showed a cytoplasmic pattern. By contrast, both wild-type and S121F with R306G localized in the cytoplasm (>95\%) or exhibited cytoplasmic and nuclear patterns. None of the cells expressing p53 exhibited any nuclear pattern. These results indicate that $\mathrm{R} 306 \mathrm{G}$ sequestered $\mathrm{p} 53$ from the nucleus to the cytoplasm. This result is reasonable since R306G is a mutation in the bipartite sequence of p53 (residues 305 and 306) as described above.

Inhibition of cell proliferation by cytoplasmically localized wild-type 553 and S121F. To examine the effect of cytoplasmically localized wild-type p53 and the S121F mutant on cell proliferation, we cultured two independent cell lines for each 


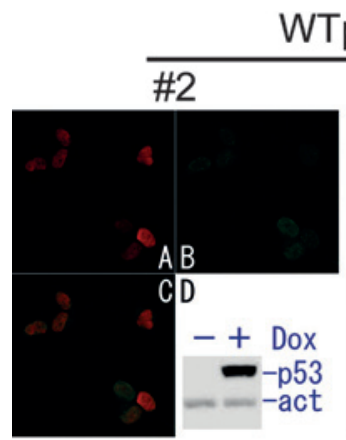

WTp53

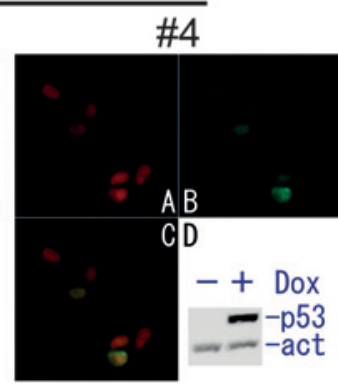

S121F

\#1
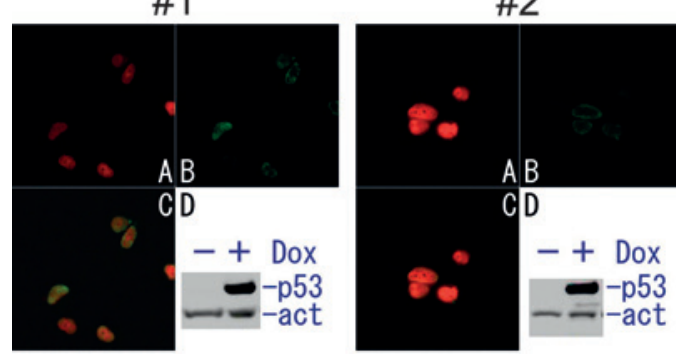
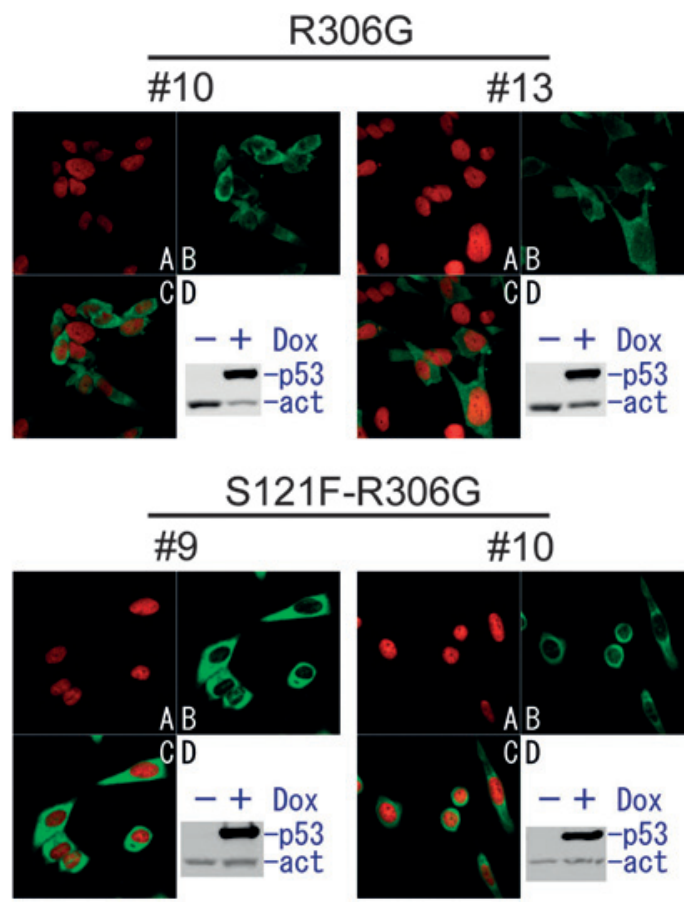

Figure 1. Inducible expression and subcellular localization of p53. Two independent clones in each category were analyzed by immunofluorescent analysis. (A) Propidum iodide; (B) p53; (C) merge of (A) and (B). (D) Western blotting of doxycycline-dependent p53 expression; $\beta$-actin was the internal control. WT, wild-type.

A

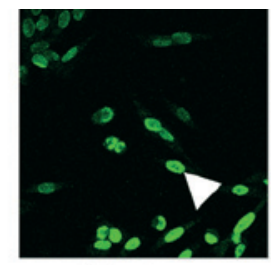

B

Nuclear

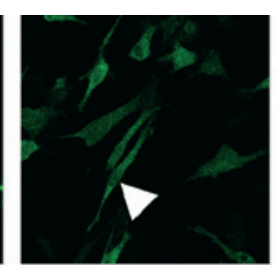

Both

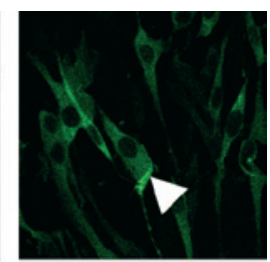

Cytoplasmic

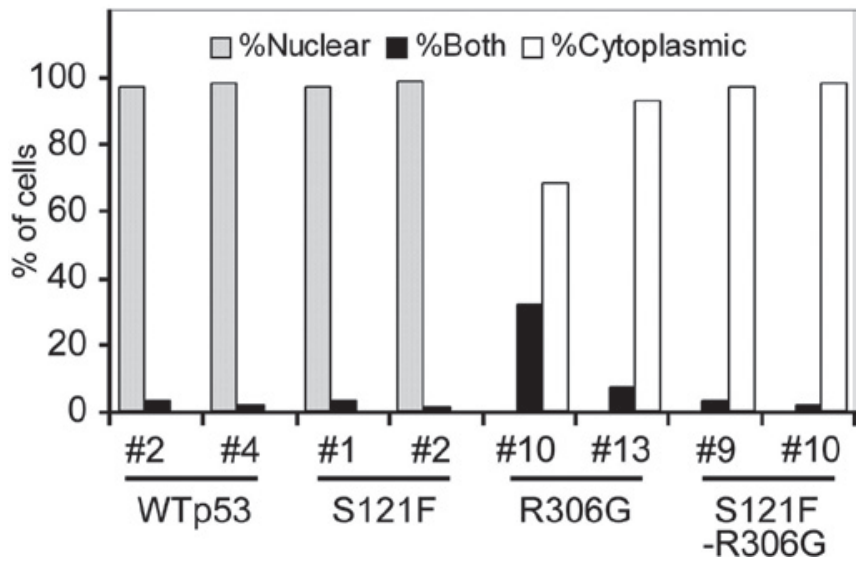

Figure 2. Quantitative analysis of the subcellular localization of wildtype p53 and S121F. (A) Subcellular localization of p53 was visualized as described in Fig. 1, and was classified as nuclear, cytoplasmic or both nuclear and cytoplasmic. (B) One hundred cells were analyzed and the percentage of each category is shown. WT, wild-type

category (wild-type, S121F, R306G, S121F-R306G and null p53) and estimated the viable cells at 24, 48 and $72 \mathrm{~h}$ after p53 induction by doxycycline (data not shown). The results at $48 \mathrm{~h}$ are shown in Fig. 3A. Although the cytoplasmic sequestration

of wild-type p53 considerably disturbed the inhibitory effect against cell proliferation by wild-type p53, some inhibitory effects remained. The cytoplasmic sequestration of S121F did not disrupt the strong inhibitory effect on cell proliferation. These results indicate a cytoplasmic function of p53 on cell proliferation in both wild-type p53 and S121F.

Induction of apoptosis by cytoplasmically localized wild-type $p 53$ and $S 121 F$. To evaluate the ability of wild-type p53 and the $\mathrm{S} 121 \mathrm{~F}$ mutant to induce apoptosis, each cell line was cultured and analyzed for a percentage of cell-cycle phase by fluorescence-activated cell sorting $24 \mathrm{~h}$ following the p53 induction by doxycycline (Fig. 3B and C). As shown in Fig. 3B, compared to the null p53 control, wild-type p53 clearly arrested cells at the G1 (49.9-59.4\%) and G2 + M (12.3-32.1\%) phases of the cell cycle and subsequently reduced the S-phase fraction (33.1-2.3\%). The sub-G1 fraction (apoptosis fraction) was only slightly increased (4.7-6.2\%) at $24 \mathrm{~h}$, whereas at $48 \mathrm{~h}$ a substantial increase was observed (1.7-16.1\%; data not shown). The cytoplasmic sequestration of p53 did not affect the cell cycle (G1, 60.5\%; G2 + M, 31.6\%), but slightly affected both the $\mathrm{S}$ phase $(5.9 \%)$ and the sub-G1 phase $(2 \%)$. S121F markedly increased the sub-G1 fraction $(44.5 \%)$ without an increase in the $\mathrm{G} 1(33.1 \%)$ and $\mathrm{G} 2+\mathrm{M}(8.3 \%)$ fractions, indicating strong apoptotic induction without cell cycle arrest. The cytoplasmic sequestration of S121F affected the sub-G1 $(26.7 \%)$, G1 (41.2\%) and G2 + M (19.6\%) fractions only partially, indicating that a strong induction of apoptosis of S121F was retained despite the cytoplasmic sequestration. These results were consistent with the results of the cell proliferation analysis and again indicated a cytoplasmic function of p53 on both cell cycle arrest and apoptosis. 

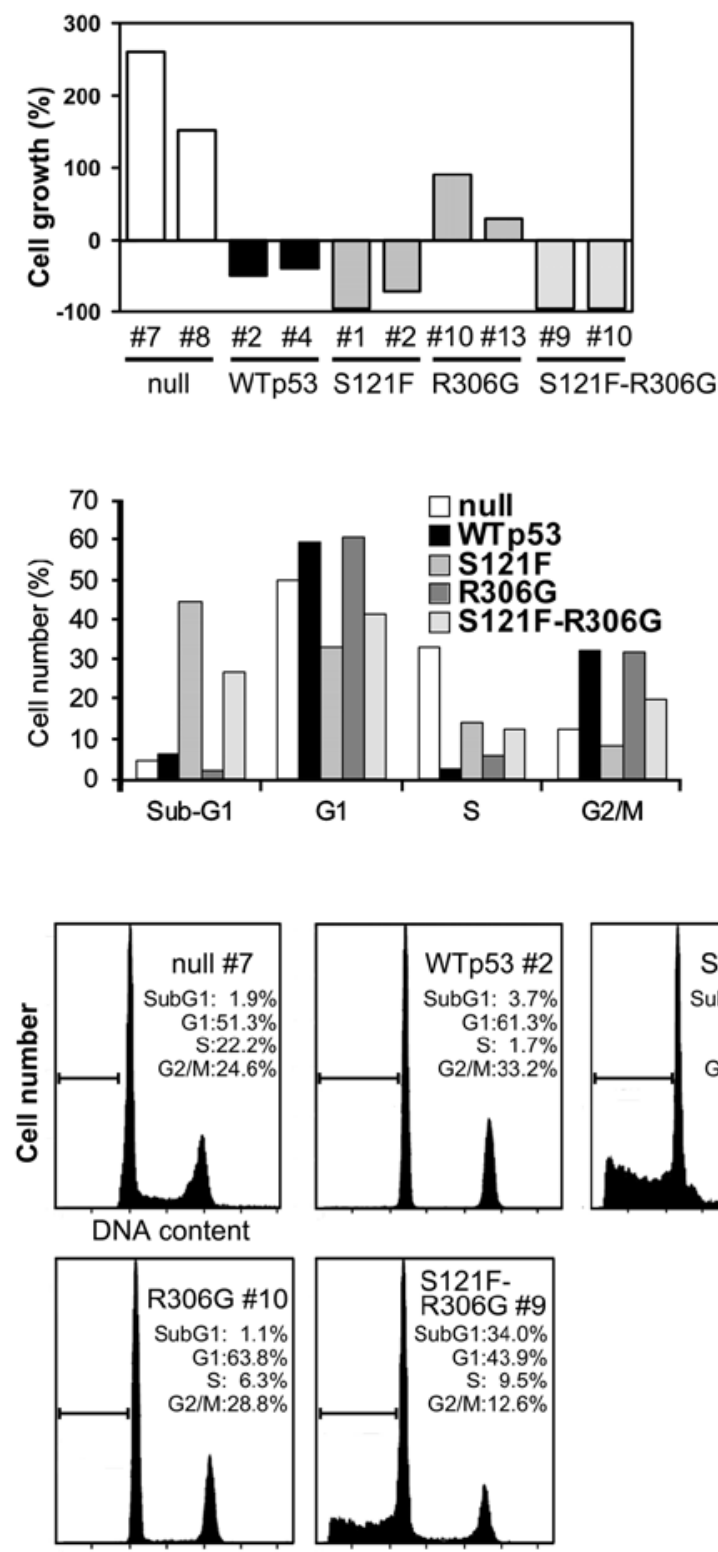

Figure 3. Inhibition of cell proliferation and induction of apoptosis by wildtype p53 and S121F. (A) Cell proliferation assays were performed at 24, 48 and $72 \mathrm{~h}$ after the addition of doxycycline. The average of triplicate data at $48 \mathrm{~h}$ is shown. (B) Cell cycle analysis by fluorescence-activated cell sorting. The average subfraction value of two independent cell lines at $24 \mathrm{~h}$ after the addition of doxycycline is shown. (C) Representative DNA histogram and subfractions at $24 \mathrm{~h}$.

Transactivation of p53 target genes by cytoplasmically localized wild-type p53 and S121F. To examine the effect of cytoplasmic sequestration on transcriptional activation by wild-type 553 and S121F, transcripts of the p53-downstream genes, MDM2, p21WAF1, BAX, NOXA, PUMA and p53AIP1, were quantitated by real-time quantitative PCR analysis at $24 \mathrm{~h}$ after p53 induction (Fig. 4). Of the six genes, all except p53AIP1 were less efficiently transactivated by S121F than by wild-type p53. The results showing a lower ability of S121F than wild-type $\mathrm{p} 53$ on transactivation were mostly consistent with our previous findings, with the exception of the result of p53AIP1 (17), which is consistent with the previous hypothesis that S121F may cause a transactivation-independent apoptotic pathway. Notably, the cytoplasmic sequestration of wild-

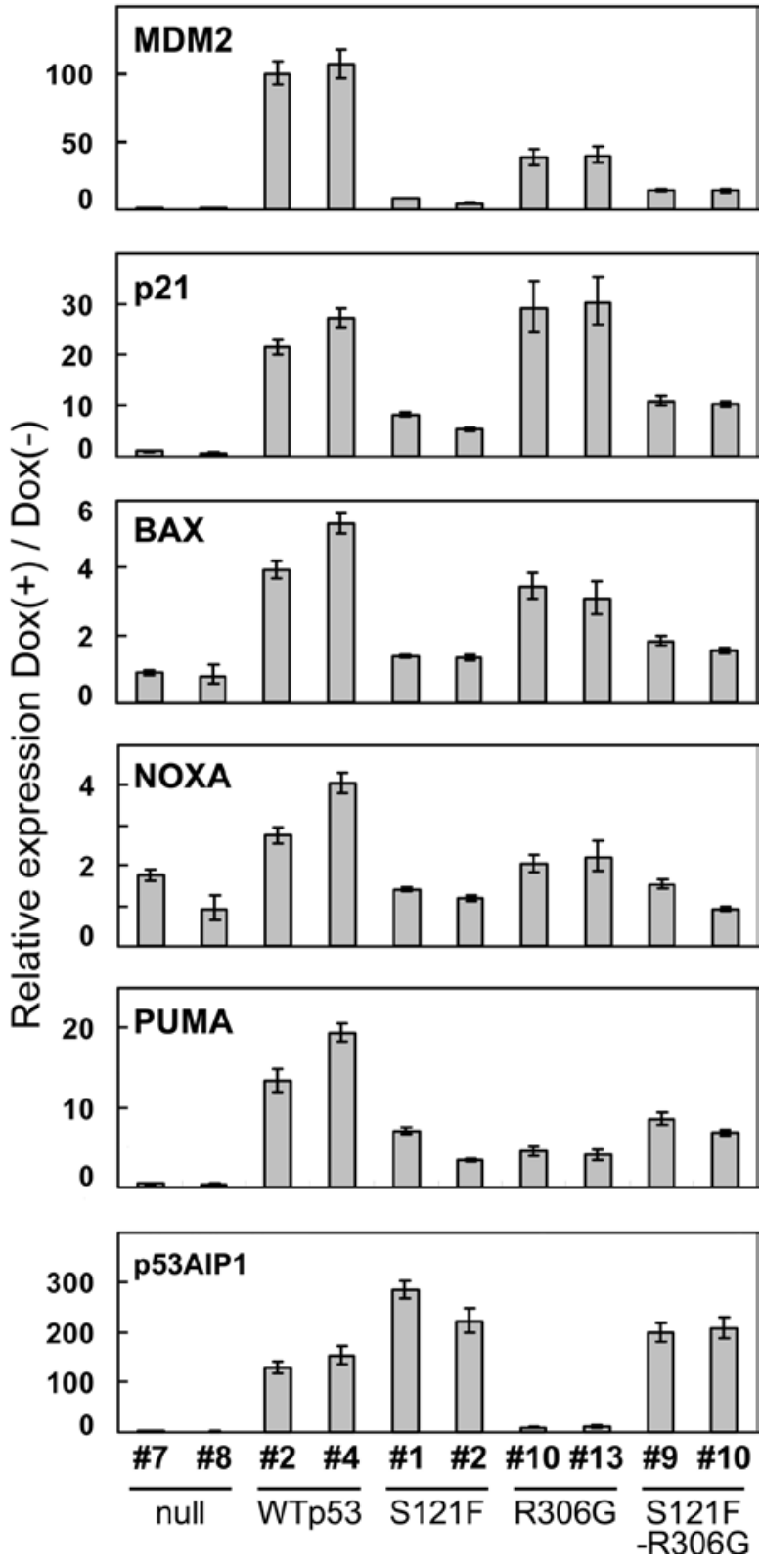

Figure 4. Transactivation of six p53 target genes by quantitative real-time PCR analysis. The expression level of each p53 target gene was collected by $\beta$-actin. Relative induced expression is shown as a ratio of the collected value of doxycycline presence against that of doxycycline absence. Two independent clones were analyzed, and the data are shown as a mean of four replicates with an error bar of standard deviation.

type p53 did not completely inactivate transactivation, but it reduced the level of transactivation in 5 of the 6 target genes (wiht the exception of $p 21 W A F 1$ ). Of note, the cytoplasmic sequestration of S121F did not change the expression profile of the target genes. The ability of cytoplasmically sequestered S121F on transactivation was also confirmed when the expression level of each p53 target gene was collected by GAPDH (data not shown).

\section{Discussion}

The cytoplasmic sequestration of p53 did not completely inactivate $\mathrm{p} 53$ function, suggesting the cytoplasmic function of p53. This finding may be the reason that mutations on the 
bipartite sequence in human tumors are extremely rare. In this context, Goldman et al showed that in a neuroblastoma cell line expressing cytoplasmically sequestered wild-type p53, p53 target genes ( $p 21 W A F 1$ and MDM2) were up-regulated following cell irradiation (18). These results also suggest that wild-type p53 retains some functional activity when it is sequestered in the cytoplasm, although p53 homologues, such as p63 and p73, may have been involved in the result. By contrast, our experimental system was a p53-specific inducible system; therefore, involvement of p53 homologue activation is unlikely.

Our previous knowledge of p53-dependent apoptosis was that after genotoxic stress, activated p53 transactivated its downstream genes in a sequence-specific manner in the cell nucleus and induced apoptosis in cells through the direct or indirect induction of the downstream protein(s); however, a transactivation-independent mechanism for p53-dependent apoptosis has been reported by several laboratories $(19,20)$. In addition, we previously indicated a lack of correlation between p53-dependent transactivation activity and the ability to induce apoptosis, and speculated that a transactivation-independent mechanism may exist (17). We excluded the nuclear function of p53, including the sequence-specific transactivation function, by introducing R306G, a mutation in the bipartite sequence at residues 305 and 306. A conditional expression system of cytoplasmically sequestered p53 was constructed and we found that cytoplasmically sequestered p53 retains its ability to arrest cell proliferation (wild-type p53) and induce apoptosis $(\mathrm{S} 121 \mathrm{~F})$. These results strongly support a cytoplasmic apoptotic function of p53. Notably, however, cytoplasmically sequestered p53 transactivated downstream genes. Therefore, we did not clarify whether cytoplasmic p53-dependent apoptosis depends on either a direct or an indirect transactivation mechanism or is independent of transactivation.

Additional experiments are required to evaluate which mechanism is crucial for p53-dependent apoptosis and to clarify the mechanism underlying super p53 (S121F)dependent apoptosis.

\section{Acknowledgements}

This study was supported by the Ministry of Education, Culture, Sports, Science and Technology (C. Ishioka and S. Kato), the Comprehensive Research and Education Center for Planning of Drug Development (CRECENDO) of Tohoku University 21st. Century COE Program (K. Yasuda and C. Ishioka), and the Gonryo Medical Foundation (C. Ishioka).

\section{References}

1. Hollstein M, Rice K, Greenblatt MS, et al: Database of p53 gene somatic mutations in human tumors and cell lines. Nucleic Acids Res 22: 3551-3555, 1994.

2. Soussi T: p53 alterations in human cancer: more questions than answers. Oncogene 26: 2145-2156, 2007.

3. Harris SL and Levine AJ: The p53 pathway: positive and negative feedback loops. Oncogene 24: 2899-2908, 2005.

4. Soussi T and Lozano G: p53 mutation heterogeneity in cancer. Biochem Biophys Res Commun 331: 834-842, 2005.

5. Moll UM, LaQuaglia M, Benard J and Riou G: Wild-type p53 protein undergoes cytoplasmic sequestration in undifferentiated neuroblastomas but not in differentiated tumors. Proc Natl Acad Sci USA 92: 4407-4411, 1995.

6. Moll UM, Riou G and Levine AJ: Two distinct mechanisms alter p53 in breast cancer: mutation and nuclear exclusion. Proc Natl Acad Sci USA 89: 7262-7266, 1992.

7. Nagpal J, Jamoona A, Gulati ND, et al: Revisiting the role of p53 in primary and secondary glioblastomas. Anticancer Res 26: 4633-4639, 2006.

8. Liang SH and Clarke MF: A bipartite nuclear localization signal is required for p53 nuclear import regulated by a carboxylterminal domain. J Biol Chem 274: 32699-32703, 1999.

9. Kim IS, Kim DH, Han SM, et al: Truncated form of importin alpha identified in breast cancer cell inhibits nuclear import of p53. J Biol Chem 275: 23139-23145, 2000.

10. Rodriguez-Lopez AM, Xenaki D, Eden TO, Hickman JA and Chresta CM: MDM2 mediated nuclear exclusion of p53 attenuates etoposide-induced apoptosis in neuroblastoma cells. Mol Pharmacol 59: 135-143, 2001.

11. Kaul SC, Deocaris CC and Wadhwa R: Three faces of mortalin: a housekeeper, guardian and killer. Exp Gerontol 42: 263-274, 2007.

12. Andrews P, He YJ and Xiong Y: Cytoplasmic localized ubiquitin ligase cullin 7 binds to 553 and promotes cell growth by antagonizing p53 function. Oncogene 25: 4534-4548, 2006.

13. Nikolaev AY, Li M, Puskas N, Qin J and Gu W: Parc: a cytoplasmic anchor for p53. Cell 112: 29-40, 2003.

14. Kato S, Han SY, Liu W, et al: Understanding the function-structure and function-mutation relationships of p53 tumor suppressor protein by high-resolution missense mutation analysis. Proc Natl Acad Sci USA 100: 8424-8429, 2003.

15. Freeman J, Schmidt S, Scharer E and Iggo R: Mutation of conserved domain II alters the sequence specificity of DNA binding by the p53 protein. EMBO J 13: 5393-5400, 1994.

16. Saller E, Tom E, Brunori M, et al: Increased apoptosis induction by $121 \mathrm{~F}$ mutant p53. EMBO J 18: 4424-4437, 1999.

17. Kakudo Y, Shibata H, Otsuka K, Kato S and Ishioka C: Lack of correlation between p53-dependent transcriptional activity and the ability to induce apoptosis among 179 mutant p53s. Cancer Res 65: 2108-2114, 2005.

18. Goldman SC, Chen CY, Lansing TJ, Gilmer TM and Kastan MB: The p53 signal transduction pathway is intact in human neuroblastoma despite cytoplasmic localization. Am J Pathol 148: 1381-1385, 1996.

19. Chen X, Ko LJ, Jayaraman L and Prives C: p53 levels, functional domains, and DNA damage determine the extent of the apoptotic response of tumor cells. Genes Dev 10: 2438-2451, 1996.

20. Haupt Y, Rowan S, Shaulian E, Vousden KH and Oren M: Induction of apoptosis in HeLa cells by trans-activation-deficient p53. Genes Dev 9: 2170-2183, 1995. 